Research Article

\title{
Research on Basketball Teaching Network Course Resource Recommendation Method Based on Deep Learning Algorithm
}

\author{
Kai Ma \\ Department of Sports, China University of Geosciences (Beijing), Beijing 100083, China \\ Correspondence should be addressed to Kai Ma; makai19990701@m.fafu.edu.cn
}

Received 18 August 2021; Accepted 1 October 2021; Published 11 October 2021

Academic Editor: Fazlullah Khan

Copyright (c) 2021 Kai Ma. This is an open access article distributed under the Creative Commons Attribution License, which permits unrestricted use, distribution, and reproduction in any medium, provided the original work is properly cited.

To solve the problem of invalid resource recommendation data and poor recommendation effect in basketball teaching network course resource recommendation, a basketball teaching network course resource recommendation method based on a deep learning algorithm is proposed. The objective function is applied to eliminate the noise in the basketball teaching network course resource data. The prominent characteristics of basketball teaching network curriculum resources are extracted using a kernel function and combined into a feature set. A convolution neural network (CNN) was employed to realize the basketball teaching network curriculum resources recommendation model. The model was assessed in terms of computation time and recognition error. To validate the performance, the proposed model was compared with two well-known recommendation models such as the learning resource recommendation method based on transfer learning and the personalized learning resource recommendation method based on three-dimensional feature collaborative domination. Experimental results show that the proposed model achieved the lowest computation time of $15 \mathrm{~s}$ and recommendation error less than $0.4 \%$ as compared with the existing model.

\section{Introduction}

With the rapid development of information technology and the continuous progress of mobile Internet technology, great changes have been taken place in human lifestyle and working state. Human life and work have become more convenient, and the progress of technology is also constantly changing our thinking and daily life activities [1]. With the development of the mobile Internet era, the popularity of various mobile terminals in people's life such as WeChat, Microblog, E-books, and network digital resources is also increasing. People can read, learn, and share information anytime and anywhere using information networks. Activities such as understanding the world and learning knowledge are no longer restricted by time and space, and the development of information technology has also brought unprecedented changes in human learning methods [2]. The core elements of the information network are information technology and digital resources. Information technology promotes the development of computer networks, and digital resources are the basis for the vigorous development of computer networks. In modern society, digital resources are the key components for information resources and have led to a revolutionary breakthrough in the field of education. The continuous development of Internet-based education, the rise of distance education, and the entry of MOOC into ordinary classroom teaching all are the products of the development of information technology [3].

As an important channel in the field of education, physical education is a highly practical and typical two-sided educational activity. In conventional physical education, the application of advanced technology and computer application is limited, which leads to the loss of student's interest in physical education [4]. In the development of information education system construction, network teaching and digital resources are added into the field of physical education, which has a great impact on the traditional teaching system of physical education. In particular, with the emergence of a large number of network digital resources such as video materials, teenagers can learn more abundant self-education sports through the network and will learn sports skills and tactics more flexibly. How to meet the challenge of 
informatization in teaching physical education and how to provide sports training to modern society have become the focus of Physical Education Reforms [5]. Basketball is a popular sport in China. It is one of the most important courses in professional sports colleges and universities. It is also the most common course in the school of physical education in China [6]. In basketball teaching, although some advanced educational technologies such as video teaching and computer courseware demonstration have been used to a certain extent, traditional classroom teaching has not changed much due to the limitations and constraints of many conditions [7]. Network teaching such as establishing a complete digital resource of basketball teaching has become a hot issue in the field of modern physical education. The digital management and application construction of basketball teaching resources has become an important process in basketball education. The recommendation of basketball teaching network curriculum resources has important guiding opinions for improving basketball learners' learning ability and skills [8]. Therefore, how to recommend basketball teaching network course resources for users has become a hot issue in the current recommendation research. Cust et al. [9] applied machine learning using neural networks and recurrent neural networks to model the training of basketball coordinate trajectories in these planes for the classification with good results for both the trained season and the season different from the one used for training. Mohammadi et al. [10] combined recurrent neural networks with weight-learning time-varying attention and developed a model that can predict different basketball events in videos. Zhu et al. [11] investigated that the application of big data techniques into basketball can significantly improve the quality of training, measure the value of players, and ensure the fairness of the game, among others. Liu et al. [12] reported that deep learning will not only change the management mode of basketball data but will also indirectly affect the level to which basketball games will develop in the future. Yao et al. [13] presented a real-time analysis system for basketball sports data based on big data and provided a theoretical foundation for enhancing the application of deep learning in basketball and also provided a theoretical orientation for the widespread use of deep learning in the field of sports. Chen and Wang [14] examined the technical features of the basketball players' in-game video and proposed a behavior analysis method based on deep learning. The basketball court and stadium marking line features were automatically extracted and keyframes of the video captured using a spatiotemporal scoring mechanism. Finally, a behavior recognition and prediction model was proposed based on an encoder-decoder framework. In order to promote basketball teaching, this paper designs a basketball teaching network course resource recommendation method based on a deep learning algorithm. The objective function is applied to eliminate the noise in the basketball teaching network course resource data. Distinct features of basketball teaching network curriculum resources are extracted using a kernel function, and a CNN was employed to develop the basketball teaching network curriculum resources, recommendation model. The model was assessed in terms of computation time and recognition error. The scientific effectiveness of the designed method is verified by experimental research.

The rest of the paper is ordered as follows. In Section 2, the data extraction and preprocessing are discussed. In Section 3, the proposed deep learning algorithm is illustrated. The results are presented in Section 4, and finally, the conclusion is given in Section 5 .

\section{Basketball Teaching Network Course Resource Data Extraction and Preprocessing}

2.1. Data Extraction. To realize the recommendation of basketball teaching network curriculum resources, it is necessary to extract the unique properties of basketball teaching network curriculum resources. Due to the availability of a large number of online basketball teaching network course resources, the feature extraction process is more difficult and complex $[14,15]$. Therefore, it is essential to extract the distinct features of basketball teaching network course resources.

Suppose the user expected basketball teaching network curriculum resource characteristics are represented as follows:

$$
T_{i}=\left\{t_{1}, t_{2}, \ldots, t_{m}\right\}
$$

where $t$ represents the desired learning resource feature vector of the user and $m$ is the desired number of resource features for basketball teaching network courses. The expected corresponding candidate basketball teaching network curriculum resource characteristics may be expressed as follows:

$$
H_{i}=\left\{h_{1}, h_{2}, \ldots, h_{n}\right\},
$$

where $h$ shows the candidate basketball teaching network course resource feature vector and $n$ represents the number of candidate basketball teaching network course resources features. Since there is a certain difference between the basketball teaching network course resources and their corresponding candidate basketball teaching network course resources, need for standardized operations, and the characteristic parameters of basketball teaching network course resources are expressed using the following equation:

$$
G_{x}=\frac{1}{n} \sum_{i=1}^{m} T_{i} \times H_{i} \int \gamma .
$$

In equation (3), $G_{x}$ represents the characteristics of the normalized basketball teaching online curriculum resources.

The feature extraction of the basketball teaching network curriculum resources also includes user's preferences. After considering the user's characteristics of the online basketball resources, the basketball teaching network course resources are recommended to the needed user. Suppose the user resource preference features can be expressed as follows:

$$
S_{i}=\left\{s_{1}, s_{2}, \ldots, s_{k}\right\}
$$

where $k$ is the number of user features. 
The preference characteristics of user resources are represented using the following equation:

$$
A=\frac{\sum S_{i}}{k \sum L_{k}}
$$

where $L_{k}$ represents the preference feature of the user resource.

At this time, the preferred characteristics of useful resources and the characteristics of online basketball teaching resources are differentiated. The feature difference between the two is processed by kernel function [16], and the feature extraction of basketball teaching network course resources is completed.

$$
U^{*}=\frac{\sum_{i=1}^{k} \sum A\left|S_{i}-H_{i}\right|}{\sum_{i=1}^{m} \beta_{y}},
$$

where $U^{*}$ is the basketball teaching network curriculum resource features and $\beta_{y}$ represents the differentiation factor.

In the feature extraction of basketball teaching network curriculum resources, the features between them are differentiated by kernel function to complete the feature extraction of basketball teaching network curriculum resources.

2.2. Data Noise Reduction. The occurrences of noisy data in the data set can significantly impact the prediction of any meaningful information. Many empirical studies have shown that noise in data set dramatically led to decreased classification accuracy and poor prediction results [17]. Because there is some noise in the feature set of basketball teaching network curriculum resources, therefore, it is necessary to preprocess the feature set of basketball teaching network curriculum resources. The noise in the basketball teaching network course resource feature set can be eliminated through the objective function, which can be computed as follows:

$$
G=\sum_{i=1}^{n} \sum_{j=1}^{m} d_{i j} h_{i j}+\beta\left(1-\sum_{i=1}^{n} d_{i j}\right) .
$$

In equation (7), $G$ represents the target function, $d_{i j}$ is the Gaussian noise data for basketball teaching network course resource feature data, $h_{i j}$ shows the basketball teaching network course resource features data white noise data, and $\beta$ represents a noise reduction factor.

In the process of noise reduction in the feature set, due to different noise levels, secondary noise reduction is also required [18] to ensure accurate extraction of basketball teaching network course resource features and can be represented as follows:

$$
U(t)=\mu \frac{1}{\sum_{i=1}^{n}\left(h_{i j} / d_{i j}\right)},
$$

where $\mu$ describes the central point of the basketball teaching network course resource feature data.

By evaluating the membership of the characteristic data of basketball teaching network curriculum resources, this study determines the unique feature set of basketball teaching network curriculum resources and completes the preprocessing of basketball teaching network curriculum resource data.

\section{Basketball Teaching Network Course Resource Recommendation Method Based on Deep Learning Algorithm}

Recently, machine learning has become more prevalent in research and has been applied in a large number of applications, including multimedia processing, image classification, video recommendation, text mining, and social network analysis. Among various machine-learning algorithms, "deep learning is widely used in these applications [19]. The explosive development and accessibility of data and the extraordinary advancement in hardware technologies have led to the advent of new studies in distributed and deep learning. Deep learning, which has its roots in conventional neural networks, considerably outperforms its predecessors. It utilizes graph technologies with transformations among neurons to develop many-layered learning models. Many of the modern deep learning methods have been presented and have revealed promising results across different kinds of applications such as natural language processing (NLP), visual data processing, speech and audio processing, and many other well-known applications [20].

The deep learning algorithm includes a variety of artificial intelligence algorithms. In this study, we employed the wavelet neural network of the deep learning algorithm to recommend the basketball teaching network curriculum resources, and the gradient descent method is used to obtain the optimal solution of the characteristic data of the basketball teaching network curriculum resources, to realize the rapid recommendation of the basketball teaching network curriculum resources [12]. The algorithm is used to determine the relationship between basketball teaching network course resource data in a large number of the same characteristics data set. First, we predict the basketball teaching network course resources which are selected by a large number of users. The basketball teaching network course resource characteristic data are used as input to the wavelet neural network, and the preliminary difference value is computed after processing through the input layer. It can be represented as follows:

$$
\begin{gathered}
U=\sum_{i=1}^{n} R_{j-2 k}<U, \\
T_{j}>+\sum_{k \varepsilon N} l-2 k,
\end{gathered}
$$

where $U$ and $k$ represent different sequences of the basketball teaching network curriculum resource feature data input, respectively.

Based on this, the preliminary predictive value is introduced to the wavelet basis function for further processing, namely: 


$$
f=\operatorname{sgn}\left[f_{j}\right]\left(\left|f_{j}\right|-t\right)
$$

where $f$ represents the time value of the wavelet basis function iteration.

Based on equation (10), the preference model of users of basketball teaching online course resources is constructed, and the recommended basketball teaching online course resources are differentiated according to the model; it can be computed as follows:

$$
W(i)=Q_{i}\left(\frac{\sum_{i=1}^{n} b f}{f}\right),
$$

where $H(i)$ represents the final output value of the differential characteristic data of the preferred data.

To further improve the recommended accuracy, the user resource preference model further improved the gradient descent method, and the result is the user preference for basketball teaching network curriculum resources, namely:

$$
q(x)=\frac{e(k)-\gamma}{p} .
$$

In equation (12), $e(k)$ represents the original basketball teaching web course resource data, $\gamma$ is the average of the original value, and $p$ shows the standard difference for the differential data characteristics.

Based on the analysis of user preference, the convolution neural network (CNN) is used to recommend the network curriculum resources of basketball teaching. $\mathrm{CNN}$ uses different convolution kernels and is widely used in many fields. CNN is a deep learning algorithm that can take in an input, assign importance weights and biases to various aspects/objects, and can differentiate one from the other. The preprocessing required in a $\mathrm{CNN}$ is much lower as compared with other classification algorithms. While in primitive methods, features are hand-engineered, with enough training. CNN can learn these features automatically.

In this study, CNN is used to make a global recommendation for basketball teaching network course resource data. The recommendation process of CNN for basketball teaching network course resources is as follows: first, the user preference model of basketball teaching online course resources is locally characterized as follows:

$$
\varnothing_{i j}=r_{i} \sqsupset v_{j},
$$

where $\varnothing_{i j}$ represents the local eigensubmatrix of the model, $r_{i j}$ shows the row vector with the highest similarity, $v_{j}$ is the column vectors of the highest similarity, and $\beth$ indicates the two-dimensional matrices.

Because the recommendation effect has a certain locality when recommending basketball teaching network curriculum resources, the importance of the resource data is confirmed in the CNN. Therefore, with the help of CNN, it is divided into the input layer, convolution layer, and output layer, and the effect of recommendation is locally characterized. Among the layers of $\mathrm{CNN}$, the convolutional layer performs a linear weighted operation [15], the convolutional core of $\mathrm{a} \times \mathrm{n}$, and the recommendation model of the basketball teaching network is expressed as follows:

$$
T_{j}(j \in p \times q)=n\left(\sum_{i \in n} R_{i} \times K+e\right),
$$

where $e$ represents the offset and $p \times q$ is the convolutional kernel size of the recommended model for the output. The basketball teaching online course resource recommendation process is shown in Figure 1.

The user preference model of basketball teaching network curriculum resources is constructed CNN, and the grid search is used to find the optimal hyperparameters for the model.

\section{Experimental Analysis}

To prove that the proposed method can well realize the recommendation of basketball teaching network curriculum resources, the experimental analysis is carried out. In the experiment, the learning resource recommendation method based on transfer learning proposed by Tang et al. [5] and the personalized learning resource recommendation method based on three-dimensional feature collaborative domination presented in [6] are selected as reference methods to highlight the effectiveness of the proposed method.

4.1. Experimental Environment. In this experiment, the resource data of the basketball teaching network course are used from the publically available Metal database, which is a multimedia teaching resource database platform. The database contains a large number of different types of resource data. In this study, 2000 pieces of basketball teaching network course resource data are selected from the Metal database, including 500 invalid data. The experimental sample data are recommended to verify the effectiveness of the recommendation method. To determine the effectiveness of experimental data statistics, we employed SPSS13.0 software to accomplish all the experimental processes. We selected the data samples of 100 people. According to the needs of users, the 100 people are divided into 4 groups to study the effectiveness of recommending basketball resource data in the 4 groups. The recommended network topology for experiment preparation is shown in Figure 2.

4.2. Experimental Index. In the experiment, the recommendation error and recommendation computation time of basketball teaching network course resource data are taken as the experimental indexes. Among them, recommendation error is an important indicator to measure the precision and accuracy of basketball teaching network course resource data recommendation. The recommendation error is computed as

$$
W=\left(\frac{1}{n-1} \sum_{i=1}^{n} y-y_{i}\right) \times 100
$$




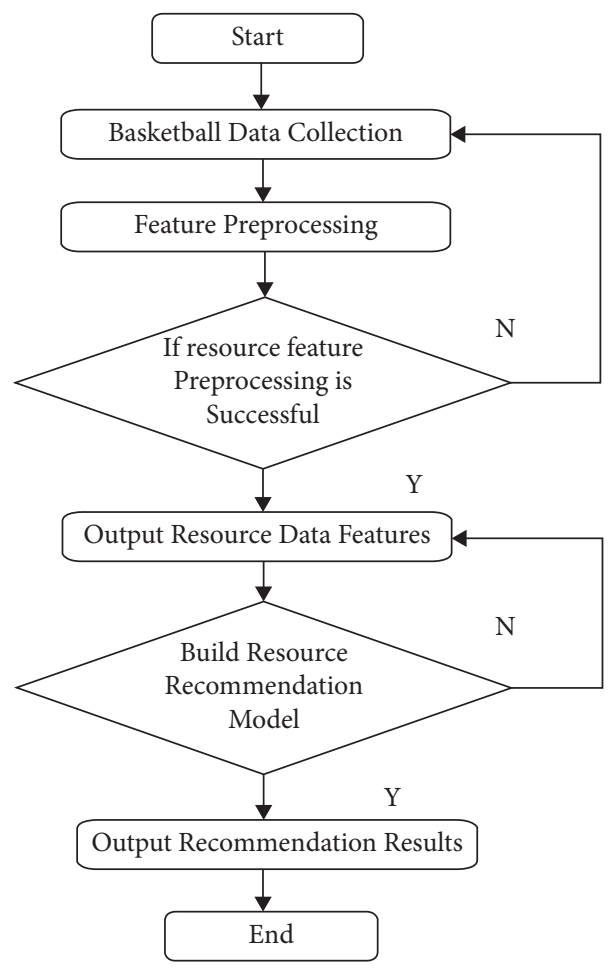

FIGURE 1: Recommendation process of basketball teaching network course resources.

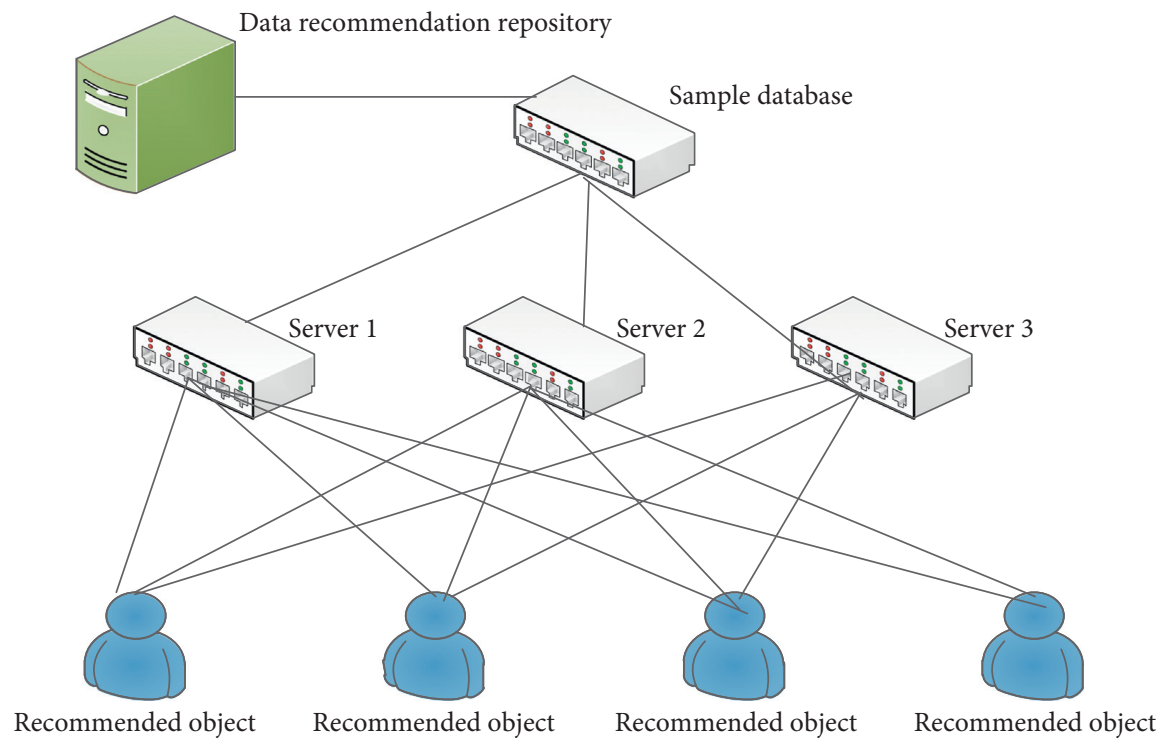

Figure 2: Recommended network topology.

where $y$ represents the recommended prediction value and $y_{i}$ shows the recommended actual value.

\section{Results}

5.1. Error Analysis. To verify the reliability of the proposed method, we compared the performance of the proposed method with that of the learning resource recommendation method based on transfer learning proposed by Tang et al.
[5] and the personalized learning resource recommendation method based on three-dimensional feature collaborative domination presented in [6]. To ensure the effectiveness of recommendation and reduce the error of recommendation of sample basketball teaching network course resource data, the three methods are iterated many times under the same experimental environment. The final recommended error for the three models is shown in Figure 3. 


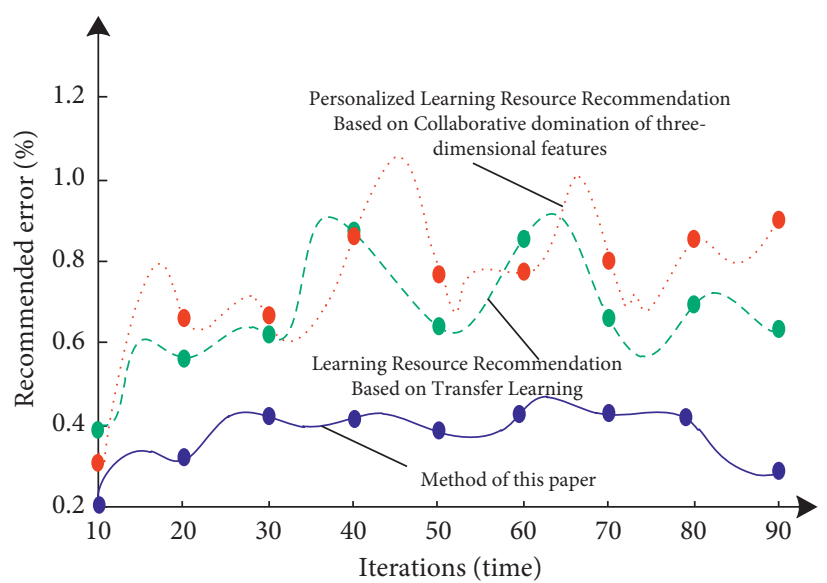

Figure 3: Comparison of recommendation error of basketball teaching network course resource data.

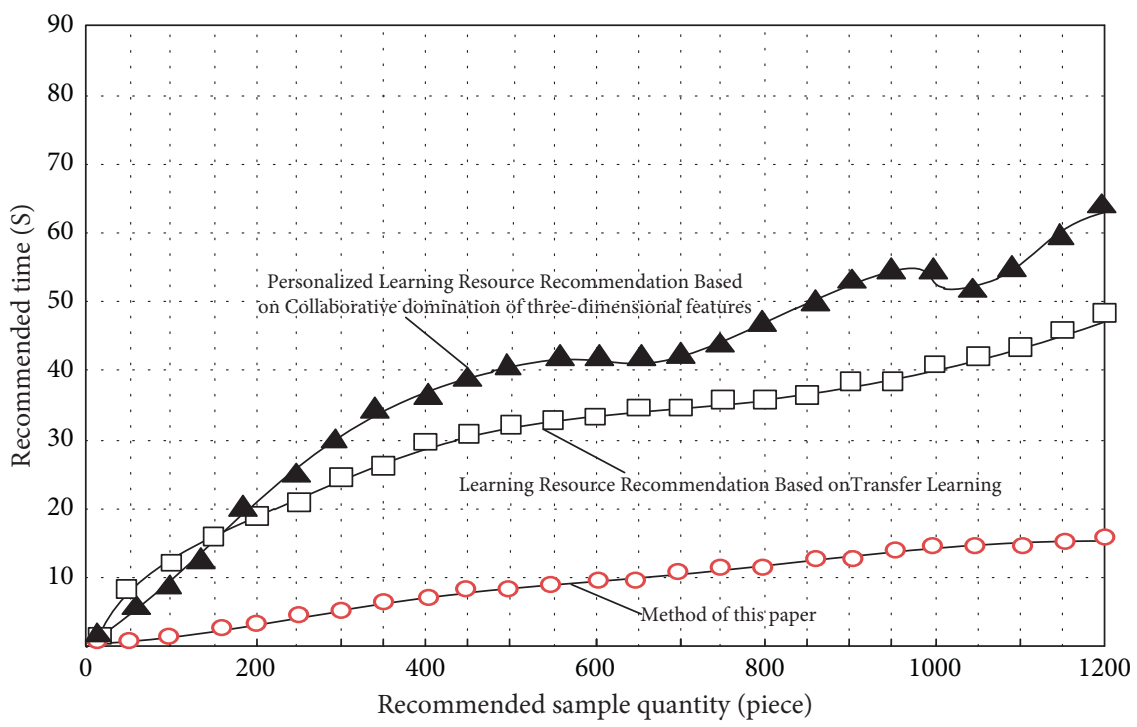

FIgURE 4: Comparison of time-consuming of basketball teaching network course resource data recommendation.

It can be seen that under the same experimental environment, the error of sample basketball teaching network course resource data recommendation by using the proposed method, the learning resource recommendation method based on transfer learning presented in [5], and the personalized learning resource recommendation method based on three-dimensional feature collaborative control in literature [6] is different. There is a certain difference in the recommended error among the three methods. Increasing the number of iteration, the error of the proposed method is almost stable and less than $0.6 \%$. When the number of iterations reaches 50 , the recommended error of this method is about $0.4 \%$ that of reference [5] is about $0.62 \%$, and of literature [6] is about $0.92 \%$, respectively. When the number of iterations is increased up to 90 , the recommended error of this method is about $0.3 \%$, that of literature [5] is about $0.61 \%$, and that of literature [6] is about $0.95 \%$. It is evident that the recommendation error of this method is the lowest because the proposed method analyzes its attributes before recommendation and uses a convolutional neural network to find the optimal solution of the recommendation model, which significantly reduces the recommendation error.

5.2. Computation Time. Computation time is the length of time required to perform a computational process. To measure the performance of the proposed system, we compared all three methods in terms of computation time. Figure 4 provides a comparison for the computation time of the proposed method with that of the learning resource recommendation method based on transfer learning and the personalized learning resource recommendation method based on three-dimensional feature collaborative domination.

It can be observed that there is a significant difference in the recommended time of the three methods. When the data samples are 600, the recommendation time of the method in this paper is about $10.1 \mathrm{~s}$, the recommendation time of the 
learning resource recommendation method based on transfer learning is about $31 \mathrm{~s}$, and the recommendation time of the personalized learning resource recommendation method based on three-dimensional feature collaborative control is about $50 \mathrm{~s}$, respectively. When the data samples were increased to 1200 , the recommendation time of the three methods is $15 \mathrm{~s}, 50 \mathrm{~s}$, and $63 \mathrm{~s}$, respectively. It is confirmed that the proposed method takes the shortest time to recommend, which verifies the effectiveness of this method. Compared with traditional recommendation methods, this method has the following advantages. First, the error of basketball teaching network course resources recommended by the proposed method proposed is the lowest, which means that the resource data recommended by this method to users are accurate. Second, the basketball teaching network course resources recommended by the method proposed in this paper are relatively short, which means that the speed of this method is relatively fast as compared with that of the other recommendation methods.

\section{Conclusion}

In this paper, a basketball teaching network course resource recommendation method based on the CNN algorithm is proposed. The objective function is used to eliminate the inherent noise in the basketball teaching network course resource feature set. With the help of the kernel function, the distinct characteristics of basketball teaching network curriculum resources are extracted and combined into a feature set. The basketball teaching network curriculum resources recommendation model is constructed with the help of a convolution neural network to realize the recommendation of basketball teaching network curriculum resources. Their model was evaluated in terms of computation time and recognition errors. To validate the performance, we compared the proposed model with two learning resource recommendation methods based on transfer learning and the personalized learning resource recommendation method based on three-dimensional feature collaborative domination. Experimental results show that the proposed model achieved the lowest computation time and recommendation error as compared with the existing models.

\section{Data Availability}

The data used to support the findings of this study are available from the corresponding author upon request.

\section{Conflicts of Interest}

The author declares that there are no conflicts of interest.

\section{References}

[1] L. Haojun, Y. Lin, and Z. Pengwei, "Method of online learning resource recommendation based on multi-objective optimization strategy," Pattern Recognition and Artificial Intelligence, vol. 32, no. 4, pp. 306-316, 2019.

[2] C. Guo, "Prediction and evaluation model of physical training for volleyball players' effect based on grey markov theory,"
Scientific Programming, vol. 2021, Article ID 6147032, 8 pages, 2021.

[3] W. Wu, R. Zhang, and L. Liu, "A personalized network-based recommendation approach via distinguishing user's preference," International Journal of Modern Physics, vol. 33, no. 6, 2019.

[4] G. M. Machado, V. Maran, and G. M. Lunardi, "A framework for an adaptive recommendation of educational resources," Computing, vol. 14, no. 3, pp. 456-452, 2021.

[5] H. Zhu, "Research on human resource recommendation algorithm based on machine learning," Scientific Programming, vol. 2021, Article ID 8387277, 10 pages, 2021.

[6] L. Hao-jun, Z. Zheng, and Z. Peng-Wei, "Personalized learning resource recommendation method based on threedimensional feature cooperative domination," Computer Science, vol. 46, no. 1, pp. 461-467, 2019.

[7] H. Li, "Dynamic facial expression recognition using sparse reserved projection algorithm for low illumination images," Scientific Programming, vol. 2021, Article ID 2658471, 10 pages, 2021.

[8] D. Zheng, Y. Zhang, and Z. Xiao, "Deep learning-driven gaussian modeling and improved motion detection algorithm of the three-frame difference method," Mobile Information Systems, vol. 2021, Article ID 9976623, 7 pages, 2021.

[9] E. E. Cust, A. J. Sweeting, K. Ball, and S. Robertson, "Machine and deep learning for sport-specific movement recognition: a systematic review of model development and performance," Journal of Sports Sciences, vol. 37, no. 5, pp. 568-600, 2019.

[10] M. Yu, J. Jin, X. Wang, X. Yu, D. Zhan, and J. Gao, "Development and design of flexible sensors used in pressuremonitoring sports pants for human knee joints," IEEE Sensors Journal, vol. 1, 2021.

[11] H. Zhu, P. Zhang, L. Wang, X. Zhang, and L. Jiao, “A multiscale object detection approach for remote Sensing images based on MSE-DenseNet and the dynamic anchor assignment," Remote Sensing Letters, vol. 10, no. 10, pp. 959-967, 2019.

[12] G. Liu, Y. Luo, O. Schulte, and T. Kharrat, "Deep soccer analytics: learning an action-value function for Evaluating soccer players," Data Mining and Knowledge Discovery, vol. 34, no. 5, pp. 1531-1559, 2020.

[13] P. Yao, "Real-time analysis of basketball sports data based on deep learning," Complexity, vol. 2021, Article ID 9142697, 11 pages, 2021.

[14] L. Chen and W. Wang, Analysis of Technical Features in Basketball Video Based on Deep the Learning Algorithm, Elsevier, Amsterdam, Netherlands, 2020.

[15] F. Jiang and X. Chen, "An action recognition algorithm for sprinters using machine learning," Mobile Information Systems, vol. 2021, Article ID 9919992, 10 pages, 2021.

[16] X. Yu, Q. Hu, H. Li, J. Du, J. Gao, and L. Sun, "Cross-domain recommendation based on latent factor alignment," Neural Computing \& Applications, vol. 29, pp. 1-12, 2021.

[17] Y. Cong, J. Yuan, and J. Luo, "Towards scalable summarization of consumer videos via sparse dictionary selection," IEEE Trans. Multimedia, vol. 14, no. 1, pp. 66-75, 2012.

[18] W. Guo and Y. Niu, "Interactive teaching system of basketball action in college sports based on online to offline mixed teaching mode," Mobile Information Systems, vol. 2021, Article ID 9994050, 10 pages, 2021.

[19] C. Li and J. Cui, "Intelligent sports training system based on artificial intelligence and big data," Mobile Information Systems, vol. 2021, Article ID 9929650, 11 pages, 2021.

[20] Q. Liu and Q. Liu, "Prediction of volleyball competition using machine learning and edge intelligence," Mobile Information Systems, vol. 2021, Article ID 5595833, 8 pages, 2021. 